\title{
Correction to: Ancient polymorphisms contribute to genome-wide variation by long-term balancing selection and divergent sorting in Boechera stricta
}

Baosheng Wang ${ }^{1,2^{*}}$ (D) Julius P. Mojica ${ }^{2}$, Nadeesha Perera ${ }^{2}$, Cheng-Ruei Lee ${ }^{3}$, John T. Lovell ${ }^{4}$, Aditi Sharma ${ }^{5}$, Catherine Adam ${ }^{5}$, Anna Lipzen ${ }^{5}$, Kerrie Barry ${ }^{5}$, Daniel S. Rokhsar ${ }^{5}$, Jeremy Schmutz ${ }^{4,5}$ and Thomas Mitchell-Olds ${ }^{2^{*}}$

\section{Correction to: Genome Biol (2019) 20:126 \\ https://doi.org/10.1186/s13059-019-1729-9}

Following publication of the original article [1], the authors reported that the Availability of data and materials section required updating. The updated text reads as follows:

The short reads of each genotype have been deposited under GenBank accession numbers SRP054739, SRP13428 3-SRP134373, SRP134393-SRP134433, SRP134436-SRP134 479, SRP134481-SRP134572 and SRP134581-SRP134671. All SNPs used in population genetic analyses, locations of all accessions, and custom scripts are available in the Dryad Data Archive at https://doi.org/10.5061/dryad.574pc6n [66]. Seeds from these accessions are available from the Arabidopsis Biological Resource Center.

\footnotetext{
Author details

${ }^{1}$ Key Laboratory of Plant Resources Conservation and Sustainable Utilization, South China Botanical Garden, Chinese Academy of Sciences, Guangzhou 510650, China. ${ }^{2}$ Department of Biology, Duke University, Box 90338, Durham, NC 27708, USA. ${ }^{3}$ Institute of Ecology and Evolutionary Biology and Institute of Plant Biology, National Taiwan University, Taipei 10617, Taiwan, Republic of China. ${ }^{4}$ HudsonAlpha Institute for Biotechnology, Huntsville, AL 35806, USA. ${ }^{5}$ Department of Energy Joint Genome Institute, Walnut Creek, CA 94598, USA.
}

Published online: 09 August 2019

\section{Reference}

1. Wang B, et al. Ancient polymorphisms contribute to genome-wide variation by long-term balancing selection and divergent sorting in Boechera stricta. Genome Biol. 2019;20:126. https://doi.org/10.1186/s13059-019-1729-9.

\footnotetext{
* Correspondence: baosheng.wang@scbg.ac.cn; tmo1@duke.edu

${ }^{1}$ Key Laboratory of Plant Resources Conservation and Sustainable Utilization, South China Botanical Garden, Chinese Academy of Sciences, Guangzhou 510650, China

${ }^{2}$ Department of Biology, Duke University, Box 90338, Durham, NC 27708, USA

Full list of author information is available at the end of the article
}

(c) The Author(s). 2019 Open Access This article is distributed under the terms of the Creative Commons Attribution 4.0 International License (http://creativecommons.org/licenses/by/4.0/), which permits unrestricted use, distribution, and reproduction in any medium, provided you give appropriate credit to the original author(s) and the source, provide a link to the Creative Commons license, and indicate if changes were made. The Creative Commons Public Domain Dedication waiver (http://creativecommons.org/publicdomain/zero/1.0/) applies to the data made available in this article, unless otherwise stated. 